\title{
Session 8: Infectious diseases
}

\author{
Wednesday 18 September 2002, Moderator: Alois Lang
}

\section{[09.30-10.00]}

Molecular dissection of the anti-HCV humoral response by phage display repertoire cloning Roberto Burioni ${ }^{\mathrm{a}}$, Nicasio Mancini ${ }^{\mathrm{a}}$, Filippo Canducci $^{\mathrm{b}}$, Antonella Grieco ${ }^{\mathrm{a}}$, Pietro E. Varaldo ${ }^{\mathrm{a}}$ and Massimo Clementi ${ }^{\mathrm{b}}$

${ }^{\mathrm{a}}$ Istituto di Microbiologia, Università di Ancona, Ancona, Italy

${ }^{\mathrm{b}}$ Università Vita-Salute San Raffaele, IRCCS Istituto Scientifico San Raffaele, Milano, Italy

Clinical and experimental evidence indicates hepatitis $\mathrm{C}$ virus $\mathrm{E} 2$ glycoprotein (HCV/E2) as the most promising candidate for the development of an effective anti-HCV vaccine. Reinfection can however occur even in the presence of vigorous production of anti$\mathrm{HCV} / \mathrm{E} 2$ antibodies. Dissection of the human immune response against $\mathrm{HCV} / \mathrm{E} 2$ showed highly variable neutralization of binding of HCV/E2 to target cells (NOB activity) among antibody clones, which probably accounts for the lack of correlation between anti-HCV/E2 titer and NOB acrivity in human sera. Identifying the human epitopes conserved among isolates and able to elicit protective antibodies would thus constitute a significant step forward. Here we describe the mapping of the B-cell epitopes present on the surface of $\mathrm{HCV} / \mathrm{E} 2$, as recognized by the immune system during infection, by the analysis of the reciprocal interactions of a panel of human recombinant Fabs derived from an HCVinfected patient. Three unrelated epitopes recognized by antibodies with no NOB activity were identified; a fourth, major epitope was defined as a clustering of minor epitopes recognized by Fabs endowed with strong NOB activity. Binding of human Fabs to HCV/E2 was not inhibited by a panel of murine monoclonal antibodies.

We also demonstrated how different human monoclonal anti-HCV/E2 Fabs differ deeply in their ability to neutralize infection of HepG2 cells by a Vesicular Stomatitis Virus pseudotype bearing HCV envelope glycoproteins.
These data can explain the lack of protection and the possibility of reinfection that occur even in the presence of a strong antiviral antibody response, providing crucial data for the design of an effective vaccine.

[10.00-10.30]

Human monoclonal antibodies specific for conformation-sensitive epitopes of $\mathrm{V} 3$ mediate cross-clade neutralization of HIV-1 primary isolates

Miroslaw K. Gorny ${ }^{\mathrm{a}}$, Constance Williams ${ }^{\mathrm{a}}$, Barbara Volsky $^{\mathrm{a}}$, Kathy Revesz ${ }^{\mathrm{b}}$, Sandra Cohen ${ }^{\mathrm{a}}$, Vicky Polonis $^{\mathrm{c}}$, William J. Honnen ${ }^{\mathrm{d}}$, Chavdar Krachmarov ${ }^{\mathrm{d}}$, Abraham Pinter ${ }^{\mathrm{d}}$ and Susan Zolla-Pazner ${ }^{\mathrm{a}, \mathrm{b}}$

${ }^{a}$ New York University School of Medicine, New York, New York 10016, USA

${ }^{\mathrm{b}}$ Research Center for AIDS and HIV Infection, Veterans Affairs Medical Center, New York, NY 100102, USA

${ }^{\mathrm{c}}$ The US Military Research Center, Rockville, MD20850, USA

${ }^{\mathrm{d}}$ Public Health Research Institute, New York, NY 100164, USA

The epitopes of the V3 domain of the HIV-1 gp120 glycoprotein have complex structures consisting of linear and conformational antigenic determinants. AntiV3 antibodies (Abs) recognize both elements but those which preferentially react to the conformational aspect of the epitopes may have more potent neutralizing activity against HIV-1, as suggested by several studies. To test this hypothesis, human anti-V3 monoclonal Abs (mAbs) were selected using a V3-fusion protein (V3-FP) which retains the native conformation of the third variable region. The V3-FP is composed of the V3 $3_{\text {JR-CSF }}$ sequence of 45 residues joined to the 263 amino acids fragment of the murine leukemia virus and expressed in CHO cells. Six human mAbs produced via fusion of a heteromyeloma with EBV-transformed peripheral blood mononuclear cells (PBMC) derived 
from HIV-1-infected individuals were selected with the V3-FP.

The mAbs recognize epitopes at the crown of the V3 loop as they competed by ELISA with biotinylated human mAb, 447-52D, specific to the tip of the $\mathrm{V} 3$ region. They were found to react more strongly with molecules displaying conformationally intact V3 than with linear V3 peptides. Treatment of gp120 with dithiothreitol and/or sodium metaperiodate reduced the reactivity with these mAbs suggesting that structures maintained by disulfide bridges and the presence of carbohydrate moieties on gp120 contribute to the epitopes recognized by the mAbs. Dependence on conformation varied among the mAbs and the reactivity of two mAbs was not significantly affected by deglycosylation of gp120451, showing some preference to linear components of the V3 epitopes, while the four mAbs which were dependent on the presence of disulfide bonds and glycosylation, can be classified as specific for conformation-sensitive epitopes. Using a virus capture assay, the mAbs showed extensive and strong cross-clade binding to native, intact virions of clades A, B, C, D and F. No binding was found with isolates from subtype E. The neutralizing activity of mAbs against primary isolates was determined in two assays: the GHOST cell assay and PHA-stimulated PBMC assay. The mAbs displayed cross-clade neutralization against primary isolates of clades A, B, C and F in both assays. Analysis by linear regression of the two variables, the binding of mAbs to native, intact virions and percent of neutralization of the same primary viruses, showed a highly significant correlation $(p<0.0001)$. The correlation indicates that the neutralization of primary isolates is strictly dependent on the potency of the anti-V3 $\mathrm{mAb}$ binding to intact virions. These studies demonstrate that human anti-V3 mAbs with preferential binding to a V3 fusion protein, which retains its native conformation, display extensive cross-clade reactivity both in binding to native, intact virions and in neutralization of primary isolates.

\section{[11.00-11.20]}

\section{Aurexis $^{\mathrm{TM}}$, a humanized monoclonal antibody} protective against $S$. aureus infection

J.T. Hutchins, D. Bryant, P. Domanski, L. Donald, A. Hall, P. Patel, B. Prater, J. Robbins, P. Syribeys, J. Vernachio, J. Wang, L. Zhang and J.M. Patti Inhibitex Inc., Alpharetta, GA 30004, USA

S. aureus is a major human pathogen that causes a spectrum of clinical conditions that range from surgical site infections to pneumonia, sepsis, and endocarditis. Due the dramatic increase in the number of communityacquired and nosocomial methicillin resistant $S$. aureus infections (MRSA), a need for novel alternative therapies exists. Microbial adhesion is recognized as the first crucial step in a series of events that leads to infection in humans. S. aureus express MSCRAMM ${ }^{\circledR}$ proteins, a family of cell surface adhesins, that mediate colonization by attaching to extracellular matrix components of host tissues or serum conditioned implanted biomaterials, such as catheters, artificial joints, and vascular grafts.

Clumping factor A (ClfA), a fibrinogen-binding MSCRAMM $^{\circledR}$ protein, has been shown to be critical virulence factor in a number of animals models. The surface expression of ClfA by virtually all $S$. aureus clinical isolates taken together with the high degree of conservation of the protein at the amino acid level suggest that it would be an ideal immunotherapeutic target. This report describes the discovery, production, characterization, and in vivo evaluation of a monoclonal antibody (mAb) against ClfA. The mAb designated 129 (Aurexis ${ }^{\mathrm{TM}}$ ) was generated via conventional immunization using a recombinant protein containing amino acids 223-559 from ClfA. Aurexis ${ }^{\mathrm{TM}}$ was first selected by ELISA reactivity with a recombinant protein containing amino acids 40-559 (A domain). The A-domain of ClfA is the functional portion of the molecule and mediates fibrinogen binding. Singlecell cloned and purified Aurexis ${ }^{\mathrm{TM}}$ has an apparent $K_{D}=1.88 \times 10^{-10} \mathrm{M}\left(K_{\text {on }} 1.43 \times 10^{6} \mathrm{M}^{-1} \mathrm{~S}^{-1}\right.$; $\left.K_{\text {off }} 2.70 \times 10^{-4} \mathrm{~S}^{-1}\right)$. Importantly, flow cytometry data indicated that Aurexis ${ }^{\mathrm{TM}}$ recognized the native ClfA MSCRAMM ${ }^{\circledR}$ protein expressed on the surface 37 clinically relevant $S$. aureus strains. Further, in in vitro assays measuring whole cell $S$. aureus binding to fibrinogen coated plates, Aurexis ${ }^{\mathrm{TM}}$ competitively inhibited $S$. aureus binding by $90 \%$ and was also able to displace $60 \%$ of $S$. aureus pre-adhered to a fibrinogen matrix. In an animal model of bacteremia, a single administration of AurexisTM protected mice against an intravenous challenge with a methicillin resistant $S$. aureus clinical isolate $(p<0.0001$ vs. murine isotype control). AurexisTM has been successfully humanized and expressed in NSO cells without loss of specificity or affinity and is under further pre-clinical evaluation. 
[11.20-11.40]

Generation of fully human monoclonal antibodies specific to bacterial and fungal pathogens in a human/mouse radiation chimera: The Trimera system

Z. Rosenthal-Galili, S. Priel, S. Shavit, S. Greenfeld, D. Terkieltaub, M. Zohar, E. Ilan, O. Ben-Moshe, A. Kischitzky, R. Eren, S. Dagan

XTL Biopharmaceuticals Ltd., Rehovot, IL, USA

The emergence of drug resistant pathogens is becoming a global concern, which requires new therapeutic approaches. Antibody therapies for treating and preventing bacterial or fungal infections constitute about $25 \%$ of the current drugs in development.

Objectives: Generation of $\mathrm{HmAbs}$ for treating and presenting GNB and Candida Albicans infection.

Methods: We have taken that approach to generate fully human monoclonal antibodies (HmAbs) directed against different pathogens such as Candida Albicans, Gram Negative Bacteria (GNB) and Staphylococcus Aureus (Staph A). These antibodies are being produced in the Trimera mouse system, which was shown previously to be a powerful tool to generate HmAbs against hepatitis $\mathrm{B}$ and hepatitis $\mathrm{C}$ viruses. This system consists of normal strains of mice that were rendered permissive for engraftment of human cells and tissues by lethal total body irradiation and radioprotection with SCID mouse bone marrow cells.

Results: We have employed the Trimera system to generate HmAbs against flagellin from GNB and against glyceraldehyde - 3-phosphate dehydrogenase (GAPDH) from Candida Albicans. Flagellin has a role in mucosal attachment and invasion and induces proinflammatory gene expression ultimately causing shock and mortality. GAPDH is a glycolitic enzyme. It is expressed on a fungal cell wall and has a role in Candida Albicans attachment to fibronectin and laminin adhesion molecules. These properties make these two molecules good candidates for immunotherapy.

In order to produce HmAbs against GNB flagellin, Trimera mice were transplanted with lymphocytes from donors positive for anti-flagellin antibodies. Immunization of these Trimera mice with the N-terminal part of flagellin presented on autologous dendritic cells resulted in amplification of the human specific immune response to GNB flagellin, up to 60 -fold over the response of the donor. Similarly, immunization of Trimera mice with Candida Albicans GAPDH resulted in amplification of the human specific immune response to GAPDH, up to 40 -fold over the response of the donor.
Conclusions: Hybridoma clones were generated from human B cells harvested from the spleens of the responding mice. These hybridomas secrete human anti- flagellin IgG and anti-GAPDH IgG. The properties of these mAbs are currently being investigated. Thus, the Trimera mouse system is an effective tool to amplify human immune responses in mice, leading to the generation of therapeutic human monoclonal antibodies.

[11.40-12.00]

Human T lymphotropic virus type 1 (HTLV-1) autoantibodies and type 1 diabetes mellitus in Jamaicans

M. Fisher Smikle

University of West Indies, Kingston, Jamaica

The clinical characteristics, autoantibody profiles and seroprevalence of human $\mathrm{T}$ lymphotropic virus type 1 (HTLV-1) were assessed in 30 Jamaican patients with type 1 diabetes mellitus. Two hundred and fifty two healthy subjects and 108 patients with Graves disease were included as controls for the HTLV-1 component of the study. The remarkable clinical data included an absence of other associated organ-specific autoimmune disease; and clinical evidence and history of congenital rubella in one patient. Islet cell cytoplasmic antibodies (ICA) were absent but $17 \%$ (5/30) of the diabetic patients tested positive for glutamic acid decarboxylase (GAD) antibodies. No other organ-specific antibodies were detected but non-organ specific autoantibodies were present in $9(30 \%)$ of the sera of diabetic patients. The seroprevalence of HTLV-1 in the patients with diabetes onset at age 15 years and older, was statistically significantly higher than in patients with Graves?disease $(5 / 21,24 \%$ v $7 / 108,6 \% ; p<0.05)$ and healthy subjects $(5 / 21,24 \% \mathrm{v} 11 / 252,4 \% ; p<0.001)$. Autoantibodies were found in the sera of $4 / 5$ (80\%) of the diabetic patients positive for HTLV-1. The likely polyaetiological nature of type 1 diabetes in Jamaicans is being further investigated at the molecular level.

[12.00-12.20]

Clinical evaluation (phase I) of a combination of two human monoclonal antibodies to HBV: Safety and antiviral properties

Rachel Eren $^{\mathrm{b}}$, Eithan Galun ${ }^{\mathrm{a}}$, Rifaat Safadic, Yaffa Ashour $^{\mathrm{c}}$, Norah Terrault ${ }^{\mathrm{d}}$, Emmet B. Keeffe ${ }^{\mathrm{e}}$, Edith Matot $^{\mathrm{a}}$, Sara Mizrachi ${ }^{\mathrm{a}}$, Dov Terkieltaub ${ }^{\mathrm{b}}$, Merav Zohar $^{\mathrm{b}}$, Ido Lubin ${ }^{\mathrm{b}}$, Judith Gopher ${ }^{\mathrm{b}}$, Daniel Shouval ${ }^{\mathrm{c}}$ and Shlomo Dagan ${ }^{\mathrm{b}}$ 
${ }^{a}$ Goldyne Savad Institute of Gene Therapy, Hadassah University Hospital

${ }^{\mathrm{b}}$ XTL Biopharmaceuticals LTD, Rehovot

${ }^{\mathrm{c}}$ Liver Unit, Hadassah University Hospital, Jerusalem, Israel

${ }^{\mathrm{d}}$ University of California at San Francisco, San

Francisco, California, USA

e Stanford University Medical Center, Stanford,

California, USA

Treatment of chronic hepatitis B virus (HBV) infection with interferon alpha and Lamivudine is characterized by lack of viral clearance, loss of response or emergence of drug-resistant mutants. Thus, new and multiple drug approaches are needed. We have developed two fully human monoclonal antibodies, directed against different epitopes of HBsAg that bind to all major HBV subtypes. A phase I clinical study was conducted to evaluate the safety, tolerability and efficacy of a mixture of these two monoclonal antibodies, $\mathrm{HBV}-\mathrm{AB}^{\mathrm{XTL}}$. A total of 27 chronic HBV patients were enrolled. In part A of the study 15 patients in 5 cohorts received a single IV infusion of antibodies with doses ranging from $0.26 \mathrm{mg}$ (260 IU) to $40 \mathrm{mg}$ (40,000 IU). All patients have completed 16 weeks of follow-up. In the second part of the study (part B), 12 patients in four cohorts received four weekly infusions of 10, 20, 40 or $80 \mathrm{mg}$ each of $\mathrm{HBV}-\mathrm{AB}^{\mathrm{XTL}}$, and were followed for additional four weeks. Administration of antibodies was well tolerated. Patients administered with doses at an Ab:Ag molar ratio of 1:2 - 1:20 showed a rapid and significant decrease in HBsAg to undetectable levels, with a correspondent reduction of HBV-DNA levels. In part $\mathrm{B}, \mathrm{HBV}-\mathrm{AB}^{\mathrm{XTL}}$ induced a significant reduction in both HBsAg and HBV-DNA levels repeatedly following administration. In conclusion, these data suggest that $\mathrm{HBV}-\mathrm{AB}^{\mathrm{XTL}}$ binds $\mathrm{HBV}$ particles and reduces serum viral titers and HBsAg levels. HBV-AB XTL could be combined with other monotherapies that are currently used to treat HBV carriers. 